\title{
MÁS QUE PALABRAS POR MINUTO: LAS OTRAS HABILIDADES QUE AFECTAN LA COMPRENSIÓN EN $1^{\circ}$ BÁSICO ${ }^{1}$
}

\author{
Daniela Vergara ${ }^{2}$ \\ Katherine Strasser ${ }^{3}$ \\ María Francisca del Río ${ }^{4}$
}

\begin{abstract}
RESUMEN
Se examinó el poder explicativo de un conjunto de habilidades sobre dos formas de medir la comprensión de textos -Comprensión Lectora (CL) y Auditiva (CA)-, en niños de $1^{\circ}$ básico. Se evaluó la contribución en la CA y CL de tres habilidades cognitivas superiores, dos medidas de conocimiento, tres medidas de función ejecutiva, la conciencia fonológica y la decodificación, con el fin de determinar el rol que cumplen en la comprensión en niños/as que están recién comenzando a decodificar. La hipótesis del estudio fue que las habilidades superiores y de conocimiento cumplirían un rol menor frente a la decodificación en esta etapa. Un total de 202 niños y niñas fueron evaluados. La regresión múltiple reveló que las variables cognitivas superiores y el conocimiento fueron más relevantes para predecir la CL que la decodificación, pero la CA fue difícil de modelar. Se analizan las implicancias para la enseñanza temprana de la lectura y el lenguaje.
\end{abstract}

Palabras clave: comprensión lectora, comprensión auditiva, predictores tempranos, monitoreo, exposición a libros, vocabulario.

\section{BEYOND WORDS PER MINUTE: THE OTHER SKILLS THAT INFLUENCE COMPREHENSION IN FIRST GRADE}

\section{ABSTRACT}

The present study examines the explanatory power of a set of skills regarding two measures of text comprehension: Listening Comprehension (LC) and Reading Comprehension (RC) in first grade students. We evaluated the contribution to $L C$ and $R C$ of three higher-order cognitive skills, two measurements of knowledge, three measurements of executive function, phonological awareness and decoding, to examine their role in the comprehension of children who are just beginning to decode. We hypothesized that higher-order cognitive skills and knowledge would have a smaller role, in comparison to decoding. Two hundred and two first graders from the Santiago metropolitan area were assessed. Multiple regression models revealed that the variables of higher-order cognitive skills and knowledge were as important and in some cases more so, than decoding in explaining the RC of these first graders. LC was difficult to model in this sample. Implications for early teaching of reading and language are discussed.

Keywords: reading comprehension, listening comprehension, early predictors, monitoring.

1 Este estudio se pudo realizar gracias al Fondo Nacional de Ciencia y Tecnología (Fondecyt), a través del proyecto $\mathrm{N}^{\circ} 1110857$.

2 Escuela de Psicología, Pontificia Universidad Católica de Chile, Santiago Chile. Contacto:dpvergarad@gmail.com

3 Escuela de Psicología, Pontificia Universidad Católica de Chile, Santiago Chile. Contacto: kstrasse@uc.cl

4 Facultad de Educación, Universidad Diego Portales, Santiago, Chile. Contacto: francisca. delrio@mail.udp.cl 


\section{Introducción}

La comprensión auditiva (CA) y comprensión lectora (CL) tienen un valor clave para el aprendizaje en la etapa escolar (Beck, McKeown $\&$ Kucan, 2002). La comprensión auditiva incluye entender instrucciones, explicaciones e historias transmitidas en forma oral. La comprensión lectora, por otro lado, implica primero decodificar las figuras gráficas hacia un formato lingüístico. En ambos casos, la comprensión consiste en construir una representación global, coherente e integrada de lo leído o escuchado (Kendeou, van den Broek, White \& Lynch, 2009; Oakhill \& Cain, 2012). Diversos estudios han mostrado que la CA y la CL tienen predictores comunes y se relacionan entre sí (Catts \& Kamhi, 2005; Potocki, Ecalle \& Magnan, 2013). Esto sugiere que comprender mejor cómo desarrollar la CA podría tener efectos positivos en la CL. Pese a ello y, a diferencia de la CL, la enseñanza explícita de la CA no es enfatizada en los currículos de países latinoamericanos (UNESCO, 2013). Evidencia adicional de esta disociación es la gran cantidad de estudios que buscan explicar la comprensión de textos escritos, en comparación con la escasez de estudios similares que ayuden a entender qué factores explican la CA en edad escolar (Florit, Roch \& Levorato, 2014). Esto representa una limitación para las posibilidades de estimular la CA en forma intencionada y explícita en la escuela.

El objetivo del presente estudio fue evaluar la contribución de variables básicas y complejas para explicar tanto la CA como la CL de niños de $1^{\circ}$ básico, que se caracterizan por presentar un nivel incipiente de decodificación. El modelo predictivo evaluado incluye variables asociadas a la decodificación (como la lectura de letras y palabras y la conciencia fonológica), y variables cognitivas superiores, metacognitivas y de conocimiento previo (razonamiento verbal, función ejecutiva, monitoreo de la comprensión, vocabulario expresivo y la exposición a libros). De estas, varias han sido asociadas con la CL en individuos mayores (Kendeou et al., 2009; Oakhill \& Cain, 2012), pero algunas no han sido examinadas en relación con la comprensión auditiva ni lectora en niños que recién comienzan a decodificar, como son los estudiantes de $1^{\circ}$ básico. Comprender la relación entre CA y CL desde edades tempranas permitirá contar 
con evidencia para diseñar intervenciones de lenguaje y lectura más adecuadas.

\section{1. ¿Qué factores explican la comprensión lectora?}

La CL representa uno de los principales factores para el éxito en la enseñanza primaria y secundaria (Nation, Cocksey, Taylor \& Bishop, 2010), así como un factor determinante en la inserción laboral de los individuos (Manzi, Strasser, San Martín \& Contreras, 2008) y el desarrollo económico de los países (Organisation for Economic Cooperation and Development, OECD, 2012). Comprender un texto escrito consiste en construir una representación coherente e integrada de su significado (Kendeou et al., 2009; Oakhill \& Cain, 2012) y, por esta razón, la comprensión de un texto va más allá de la comprensión de sus frases componentes. Para construir una interpretación global de un texto es necesario integrar las distintas partes del mismo, al tiempo que se asocia lo que se lee con el conocimiento del mundo por medio de inferencias y conexiones lógicas.

De acuerdo con el modelo simple de la lectura (Hoover $\&$ Gough, 1990), la CL es función de dos factores principales: la comprensión del lenguaje oral y la capacidad de decodificación. Es decir, en una primera instancia se requiere comprender el significado de las letras y las palabras, para luego poder utilizar el conocimiento de mundo y los procesos mentales superiores que permiten la construcción del significado. Por esta razón, una baja capacidad de reconocer letras y palabras resultará en una pobre CL, pero una buena capacidad de lectura de letras y palabras no necesariamente asegura una buena CL. Que la lectura de letras y palabras no garantiza la comprensión queda demostrado por los múltiples estudios que encuentran que existen niños con buena fluidez, pero que no logran una adecuada comprensión (Cain \& Oakhill, 2006; Canet-Juric, Burin, Andrés y Urquijo, 2013). Estos últimos han demostrado que, a medida que se alcanza buena velocidad, la influencia de otros factores cognitivos y de lenguaje sobre la CL cobra mayor importancia (Tighe \& Schatschneider, 2014; Tilstra, McMaster, van den Broek, Kendeou \& Rapp, 2009). Así, mientras que en los lectores incipientes el conocimiento de las letras y la conciencia fonológica explican 
gran parte de las diferencias en CL (Aikens \& Barbarin, 2008), en decodificadores experimentados estas se explican principalmente por procesos como las inferencias (Eason, Goldberg, Young, Geist $\&$ Cutting, 2012), la metacognición (Andreassen \& Braten, 2010; Eason et al., 2012), el conocimiento previo (Kendeou, Rapp \& van den Broek, 2003), el vocabulario (Ouellette $\&$ Beers, 2010), el razonamiento verbal (Tighe $\&$ Schatschneider, 2014) y la función ejecutiva (Andreassen $\&$ Braten, 2010; Berninger et al., 2010).

Sin embargo, ¿cuál es el rol de esas habilidades cognitivas y lingüísticas superiores en la CL de niños cuya lectura de palabras no es aún automatizada? La mayoría de los estudios en lectores incipientes se centran en habilidades de decodificación, y pocos investigan procesos de pensamiento y lenguaje más complejos. La edad más temprana en la que se ha estudiado la interacción entre procesos de decodificación y superiores para explicar la CL es aproximadamente al nivel de $3^{\circ}$ básico. Oakhill y Cain, demostraron que habilidades complejas como realizar inferencias, monitorear la comprensión, el conocimiento previo y el conocimiento de la estructura de las historias, cuando son medidas entre los 9 y 10 años, predicen la CL unos años después, luego de controlar por decodificación y habilidad verbal (Cain, 2006; Cain, Oakhill \& Bryant, 2004; Oakhill \& Cain, 2012; Oakhill, Hartt \& Samols, 2005).

En el idioma español, Canet-Juric y otros (2013) estudiaron varias de estas variables en relación con la CL de niños de ocho y nueve años. Los resultados mostraron que los buenos y malos comprendedores diferían en su habilidad de monitoreo, de hacer inferencias, en su vocabulario, memoria de trabajo y control inhibitorio. Otra variable superior que ha sido estudiada en relación con la lectura es el contacto con libros (Davidse, DeJong, Bus, Huijnregts \& Swaab, 2011; Mol, Bus, \& de Jong, 2009; Mol, Bus, de Jong, \& Smeets, 2008). Los estudios son consistentes en mostrar que los niños que conocen más textos escritos tienen mejor decodificación y también mejor CL, además de un mejor lenguaje oral.

Lo anterior sugiere que las habilidades cognitivas y lingüísticas complejas, así como las experiencias con libros, son relevantes desde 
temprano en la comprensión de textos escritos. Pero ¿desde qué tan temprano? Las investigaciones citadas estudian niños a partir de los ocho o nueve años, edad en la que un porcentaje importante ya domina la decodificación, pero es posible que los procesos complejos ejerzan su efecto desde más temprano, cuando aún no se ha logrado un mínimo de velocidad lectora. El primer objetivo del presente estudio es examinar si estos procesos influyen en la CL de lectores muy incipientes, donde la decodificación aún no ha sido automatizada.

\section{2. ¿Qué factores explican la comprensión auditiva?}

Existe evidencia que muestra que los predictores de la CL arriba descritos también afectan la comprensión del discurso no escrito, como textos auditivos, libros mudos, o videos (Florit et al., 2014; Potocki et al., 2013). Diferentes estudios han mostrado que tanto la CL como la CA utilizan algunos procesos lingüísticos y cognitivos similares (Catts \& Kamhi, 2005; Florit et al., 2014). Entre estos, Lehto y Anttila (2003) encontraron que en niños de $2^{\circ}$ y $6^{\circ}$ grado existía un patrón similar de desarrollo entre la CA y la CL. En forma similar, Garner y Bochna (2004) hallaron que niños de $1^{\circ}$ grado que recibieron enseñanza explícita acerca de la estructura de las historias escritas mejoraron su comprensión de historias escuchadas en comparación con un grupo control, mostrando transferencia entre ambos procesos. Complementariamente, estudios con preescolares no lectores muestran que el uso de inferencias, la memoria de trabajo, vocabulario y el razonamiento verbal, contribuyen todos al rendimiento en tareas de CA (Florit, Roch $\&$ Levorato, 2011; Florit et al., 2014; Florit, Roch, Altoè \& Levorato, 2009). En idioma español y, trabajando con un cuento sin palabras en lugar de CA, Strasser y del Río (2014) demostraron que la comprensión de un libro sin palabras en niños de kínder estaba explicada por su nivel de vocabulario, capacidad de monitoreo e inferencias, una vez que se controlaba por su memoria de trabajo y edad.

De esta forma, la evidencia revisada sugiere que la CA y CL utilizan procesos lingüísticos y cognitivos similares, en prelectores y lectores fluidos. Esto abre una interesante posibilidad instruccional para los docentes de preescolar y de los primeros grados de primaria: 
utilizar medios no escritos para estimular las habilidades cognitivas y lingüísticas que potenciarán a futuro la CL. Trabajar en $1^{\circ}$ de primaria únicamente con textos que estimulan la decodificación, limita severamente el grado en que se pueden ejercitar habilidades complejas como el monitoreo, el razonamiento, o las inferencias. En cambio, trabajar con videos, libros mudos, o historias escuchadas, que estimulan la CA, abre la posibilidad de utilizar otros materiales para fortalecer habilidades que impactarán más tarde en la CL.

Para poder afirmar que este trabajo incidirá en la CL, es conveniente primero examinar el grado en que esas habilidades explican ambos formatos de comprensión del discurso (CA y CL). Así, además de conocer si estas explican la CL de lectores incipientes, un segundo objetivo de este estudio fue identificar el grado en que la CL y la CA son explicadas por el mismo conjunto de variables.

Debido a la gran variabilidad en la fluidez y velocidad de la decodificación en lectores incipientes, se hipotetizó que la CL en primer grado se explicaría en mayor medida por las variables de decodificación y conciencia fonológica, pero que también se observaría una contribución, aunque pequeña, de variables complejas como el razonamiento verbal, capacidad de inferencia, el conocimiento de los libros, y el monitoreo de la comprensión. Por su parte, una segunda hipótesis planteó que para explicar la CA tendrían un mayor peso las variables complejas antes mencionadas y, un menor peso, las relacionadas de decodificación y conciencia fonológica. Por último, una tercera hipótesis planteó que variables cognitivas y de conocimiento, como la función ejecutiva, la exposición a libros y el vocabulario, influirían en forma similar en la CL y en la CA de niños de $1^{\circ}$ de primaria.

\section{Metodología}

\subsection{Participantes}

Esta investigación se realizó con los participantes de un estudio mayor reclutados en kínder. Fueron 257 niños y niñas (108 de sexo femenino) los evaluados en kínder, de los cuales 202 fueron evaluados 
nuevamente en $1^{\circ}$ básico (78 mujeres), con un promedio de edad de 6,56 años. El desbalance de sexos en la muestra original (kínder) se debió a los estudiantes de quienes se recibió consentimiento, lo cual empeoró con el abandono selectivo del estudio de estudiantes de sexo femenino entre kínder y primero. Los niños pertenecían a ocho establecimientos de la Región Metropolitana de Santiago de Chile, siete de los cuales eran particulares subvencionados, y uno particular pagado. Según la clasificación socioeconómica del Ministerio de Educación de Chile, los siete primeros pertenecían al grupo C (medio), y el octavo perteneciente al grupo D (medio-alto). Para los análisis se los dividió en cuatro categorías, de acuerdo con el copago que deben hacer los apoderados, de manera de controlar por nivel socioeconómico. Debido a la alta segregación socioeconómica de las escuelas chilenas, aproximar el nivel socioeconómico de los niños de acuerdo con la escuela a la que asisten resulta apropiado (Mizala \& Torche, 2012).

\subsection{Instrumentos}

Se aplicaron dos pruebas de comprensión, una de CL y otra de CA como variables dependientes del estudio. Como variables predictivas se evaluaron tres habilidades cognitivas superiores (capacidad de hacer inferencias, razonamiento verbal y monitoreo de la comprensión); dos medidas de conocimiento (exposición a libros y vocabulario); tres medidas de función ejecutiva (control inhibitorio, flexibilidad cognitiva y memoria de trabajo) y dos medidas asociadas a la lectura de letras y palabras (decodificación y conciencia fonológica). Además, se controló por edad, sexo de los niños y por los días transcurridos desde el inicio del año escolar al momento de la evaluación.

En total se utilizaron 12 instrumentos. A continuación se detallan, organizados de acuerdo con la variable que buscan medir.

\section{a. Comprensión de lectura}

Se utilizó el Test LEE (test de lectura y escritura en español) (DefiorCitorel, et al., 2006). Esta prueba incluye tres pasajes (dos narrativos, uno expositivo). Para cada pasaje los niños deben responder a ocho preguntas (seis abiertas y dos de selección múltiple). Las preguntas 
se hacen en voz alta y se les permite revisar los textos si lo desean. El alfa de Cronbach de las 24 preguntas, calculado a partir de los datos propios, alcanzó a 0,64. La prueba no se aplicó en los casos en los que no se alcanzaba un nivel mínimo de decodificación. La prueba no tiene normas para la población chilena, solo para poblaciones argentinas y españolas. Se utilizó el puntaje bruto, controlando por la edad de los niños.

\section{b. Comprensión auditiva}

La comprensión auditiva se midió con una versión adaptada y traducida de la prueba Test of Story Comprehension (Petersen \& Spencer, 2012). Esta prueba consiste en 40 historias cortas (aproximadamente de 70 palabras cada una) con la misma estructura (el protagonista tiene una meta, enfrenta un problema, busca alternativas y, finalmente, logra solucionar la situación, enfrentando diferentes emociones a lo largo de la historia). Se tradujeron y pilotearon varias de las historias y se escogieron tres para administrar en el estudio final. A los niños se les hicieron seis preguntas acerca de cada historia relativas a los personajes, problemas, soluciones y emociones. Cada respuesta recibió 0,1 o 2 puntos, según el dominio de estos elementos. La consistencia interna de las 18 preguntas en la aplicación (alfa de Cronbach) alcanzó 0,73. Esta prueba fue fácil para los niños, quienes lograron muchas veces un efecto de techo.

\section{c. Decodificación}

Este puntaje se creó agregando dos mediciones. Para la primera se utilizó la Prueba de identificación de letras y palabras de la Batería III Woodcock-Muñoz, que tiene una confiabilidad reportada por el editor de 0,91 (Muñoz-Sandoval, Woodcock, McGrew \& Mather, 2005). La segunda medición es velocidad de lectura, la que se calculó a partir de la prueba LEE, tomando el tiempo que los niños demoraron en leer cada uno de los cuentos y dividiendo ese número por la cantidad de palabras leídas. Para agregar las dos pruebas, estas se transformaron a puntaje estándar y se las promedió. La correlación entre ambas variables fue de $0,588(p<, 01)$. 


\section{d. Conciencia fonológica}

Se utilizaron dos subescalas (rimas y cancelación) de la Prueba de discernimiento de sonidos de la Batería III Woodcock-Muñoz (consistencia interna reportada por el editor de 0,81) (Muñoz-Sandoval et al., 2005). La prueba de rimas solicita a los niños que seleccionen y luego produzcan una palabra que rime con la palabra objetivo. La prueba de cancelación solicita al niño que indique qué palabra se forma si se cancela una sílaba y luego un fonema de una palabra. Los puntajes fueron transformados a puntaje $\mathrm{Z}$ y promediados. La correlación entre ambas variables fue de $0,413(p<0,01)$.

\section{e. Vocabulario}

Para medir esta habilidad se utilizó el Test de inteligencia para niños de Wechsler WISC III, subprueba de vocabulario. Esta escala pide al niño entregar definiciones de palabras y se le asigna a cada palabra un puntaje de 0, 1 o 2 puntos, dependiendo de la información y estructura de la definición proporcionada. Esta prueba tiene un alfa de Cronbach reportada por el editor de 0,79 (Ramírez y Rosas, 2007).

\section{f. Razonamiento verbal}

También se utilizó una subescala del Test de inteligencia para niños de Wechsler WISC III, la subprueba de analogías. Esta prueba solicita al niño indicar en qué se parecen dos palabras y se le asigna a cada una un puntaje de 0,1 o 2 puntos, dependiendo de la información y estructura de la explicación proporcionada. Esta prueba tiene un alfa de Cronbach reportado de 0,74 (Ramírez y Rosas, 2007).

\section{g. Inferencias}

Para medir la capacidad de realizar inferencias se adaptó una tarea desarrollada por Oakhill (1982). La prueba consiste en 10 microcuentos de dos frases cada uno. Cada microcuento admite una inferencia válida (por ejemplo, en el microcuento "El soldado disparó al blanco; el blanco era un árbol", la inferencia válida es que el soldado disparó al árbol). Los niños escuchan los 10 microcuentos, luego de lo cual se les pide que escuchen 30 frases y que juzguen, para cada una, si estaba entre las frases originales escuchadas (la 
pregunta es "iyo dije que...?"). De las tres frases que deben evaluar los sujetos, una es una frase textual del microcuento (" $i$ Yo dije que el soldado disparó al blanco?"), otra consiste en una inferencia válida (“¿Yo dije que el soldado disparó al árbol?”) y, la última representa una inferencia incorrecta ("iYo dije que el soldado estaba sentado?"). Oakhill demostró que las personas con tendencias a formar inferencias tienden a cometer más errores del tipo escoger inferencias válidas que no fueron escuchadas, mientras que las personas con menor tendencia a hacer inferencias tienden a cometer cualquiera de los dos errores posibles (inferencia válida o incorrecta). Por ende, se construyó un puntaje sumando las frases correctamente recordadas (textuales) con las inferencias válidas recordadas, restando las frases no válidas. Por la naturaleza del puntaje, no se puede calcular la consistencia interna.

\section{h. Monitoreo de la comprensión}

Se construyó un cuento al que se le introdujeron errores. El cuento fue leído al niño con la instrucción de indicar los errores. Cada vez que el niño identificaba un error debía indicarlo a la examinadora y justificarlo. Se asignó un punto por cada error identificado y correctamente justificado. El cuento iba acompañado de siete láminas y tiene un total de quince errores, teniendo un máximo de 15 puntos. El alfa de Cronbach de esta prueba fue de 0,79.

\section{i. Función ejecutiva}

Se utilizaron diferentes pruebas según la habilidad que se buscaba medir.

- Memoria de trabajo. Se utilizaron dos pruebas de la Batería de memoria de trabajo de Pickering y Gathercole (2001) (dígitos inversos y series de palabras). La prueba de dígitos inversos requiere repetir en forma inversa una serie de números. La prueba de series de palabras, en tanto, requiere repetir una serie de palabras. Para construir la escala de memoria de trabajo se promediaron los puntajes $\mathrm{Z}$ de las dos pruebas.

- Control inhibitorio. Tarea de la cuerda floja (Kochanska, Murray, Jaques, Koenig, \& Vandegeest, 1996). En esta tarea se solicita al niño que camine por una línea marcada en el piso y se toma el tiempo. Luego se le solicita que realice la 
misma tarea pero más lento, y finalmente "lo más lento que puedas". El puntaje es el porcentaje de reducción en el tiempo relativo al tiempo base.

- Flexibilidad cognitiva. Tarea de algo es igual (Willoughby, Wirth \& Blair, 2012). En esta tarea se le muestran al niño tres estímulos, y se le señala que dos de ellos son iguales en una dimensión (por ejemplo, color). Luego se le muestra un cuarto estímulo que es similar a otro en una nueva dimensión, y se le pide que indique a cuál estímulo se parece. El alfa de Cronbach del puntaje colapsado de esta prueba fue de 0,62.

\section{j. Exposición de libros infantiles}

Prueba de reconocimiento de portadas con 41 libros infantiles tomados del plan lector chileno, títulos populares y clásicos. Se les mostraba a los niños un póster con estas portadas, donde se eliminaron los títulos y se les pedía señalar los libros que conocían (no necesariamente debían haberlos leído). Bastaba que comentaran que conocían los libros, los tenían en casa, los habían visto en la biblioteca, o que habían tenido contacto con los ellos. Esta prueba presentó un alfa de Cronbach de 0,70.

\subsection{Procedimiento}

Los directores de las escuelas fueron contactados para pedir su autorización. Los apoderados de los niños fueron contactados en una reunión organizada por el colegio. Se explicaron los procedimientos del estudio y se solicitó su consentimiento para la participación de sus niños. Los niños fueron evaluados, una vez que asintieron a participar, por estudiantes universitarias en tres sesiones, de aproximadamente de 30 minutos con cada niño evaluado, en dependencias de la escuela.

\subsection{Análisis}

Se pusieron a prueba dos modelos de regresión múltiple de mínimos cuadrados, uno para cada variable dependiente (CL y CA). La regresión múltiple se condujo con el método introducir, en un solo bloque con todos los predictores a la vez, para determinar la contribución única de cada uno. Los predictores de ambas regresiones fueron los mismos: 
decodificación, conciencia fonológica, vocabulario, razonamiento verbal, inferencias, monitoreo, función ejecutiva y exposición a libros. Además, se controló por sexo, copago de los padres, edad y días en la escuela al momento de la evaluación.

\section{Resultados}

Las Tablas 1 y 2 presentan los estadísticos descriptivos y correlaciones entre las variables del estudio.

Tabla 1. Estadísticos descriptivos

\begin{tabular}{|c|c|c|c|c|c|}
\hline \multicolumn{2}{|c|}{ Factor } & Mín. & Max. & Media & Desv. típ. \\
\hline \multicolumn{2}{|c|}{ Mujer } &, 00 & 1,00 & .39 & .49 \\
\hline \multicolumn{2}{|c|}{ Copago } & 1,00 & 4,00 & 1,95 & 1,16 \\
\hline \multirow{3}{*}{$\mathrm{CL}$} & $\mathrm{CL}$ & 13,00 & 43,00 & 29,89 & 6,13 \\
\hline & Edad (meses) & 69,00 & 92,00 & 82,07 & 3,76 \\
\hline & Días transcurridos & 166,00 & 230,00 & 188,59 & 16,28 \\
\hline \multirow{3}{*}{ CA } & CA & 49,00 & 1296,00 & 778,41 & 266,77 \\
\hline & Edad (meses) & 66,00 & 88,00 & 78,48 & 3,74 \\
\hline & Días transcurridos & 53,00 & 111,00 & 77,95 & 10,27 \\
\hline \multicolumn{2}{|c|}{ Conciencia fonológica } & $-1,40$ & 2,88 &, 09 &, 84 \\
\hline \multicolumn{2}{|c|}{ Decodificación } & $-1,25$ & 3,02 &, 11 & 90 \\
\hline \multicolumn{2}{|c|}{ Razonamiento verbal } & 3,00 & 15,00 & 11,13 & 2,60 \\
\hline \multicolumn{2}{|c|}{ Vocabulario } & 1,00 & 19,00 & 10,88 & 3,23 \\
\hline \multicolumn{2}{|c|}{ Inferencias } & 6,00 & 24,00 & 18,47 & 2,57 \\
\hline \multicolumn{2}{|c|}{ Monitoreo } &, 00 & 14,00 & 8,29 & 3,30 \\
\hline \multicolumn{2}{|c|}{ Flexibilidad cognitiva } & $-1,00$ & 1,00 &, 24 & 97 \\
\hline \multicolumn{2}{|c|}{ Control inhibitorio } &,- 03 & 7,41 &, 83 & .76 \\
\hline \multicolumn{2}{|c|}{ Memoria de trabajo } & $-2,22$ & 2,93 &, 11 &, 85 \\
\hline \multicolumn{2}{|c|}{ Exposición a libros } &, 00 & 16,00 & 4,89 & 2,49 \\
\hline
\end{tabular}

Nota: ${ }^{*} p<0,05{ }^{* *} p<0,01$. 


\begin{tabular}{|c|c|c|c|c|c|c|c|c|c|c|c|c|c|c|c|c|c|c|c|c|c|}
\hline $\begin{array}{l}\text { 声 } \\
\text { 妏 } \\
\end{array}$ & & & & & & & & & & & & & & & & & & & & & 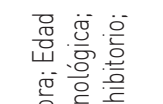 \\
\hline 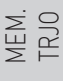 & & & & & & & & & & & & & & & & & & & & & 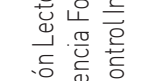 \\
\hline 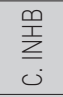 & & & & & & & & & & & & & & & & & & - & 些 & & 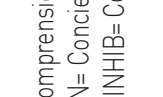 \\
\hline $\begin{array}{l}8 \\
\text { ठ } \\
\text { म } \\
\end{array}$ & & & & & & & & & & & & & & & & & - & कo & 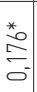 & & 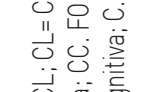 \\
\hline$\frac{5}{\bar{c}}$ & & & & & & & & & & & & & & & & - & : & 兽 & 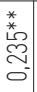 & & 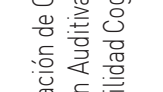 \\
\hline 岀 & & & & & & & & & & & & & & & 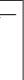 & $\begin{array}{l}0 \\
0 \\
0 \\
0 \\
1\end{array}$ & ठิ & 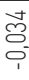 & $\begin{array}{l}\widetilde{\Xi} \\
\text { - } \\
1\end{array}$ & & 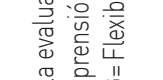 \\
\hline $\begin{array}{l}\frac{m}{0} \\
\text { ర }\end{array}$ & & & & & & & & & & & & & & - & 8 & 莣 & $\begin{array}{l}\text { aे } \\
\text { o. }\end{array}$ & 蕉 & $\begin{array}{l}\infty \\
\stackrel{0}{0} \\
0\end{array}$ & 营 & 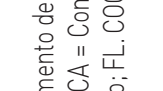 \\
\hline 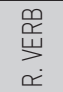 & & & & & & & & & & & & $\gamma$ & & : & E. & 莣 & 䓠 & 吾 & 莡 & 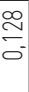 & 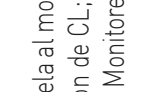 \\
\hline $\begin{array}{l}\text { 음 } \\
\text { 㟧 }\end{array}$ & & & & & & & & & & & - & 先 & & : & $\begin{array}{c}\infty \\
\text { D. } \\
\\
\end{array}$ & $\begin{array}{l}\stackrel{*}{\Xi} \\
\stackrel{\sigma}{\sigma}\end{array}$ & ఫे & 冓 & 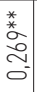 & - & 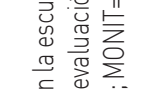 \\
\hline $\begin{array}{l}z \\
\text { z } \\
\text { j }\end{array}$ & & & & & & & & & & - & 恙 & 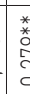 & & 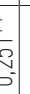 & E্ & \begin{tabular}{l}
$*$ \\
\multirow{*}{*}{} \\
$\vdots$ \\
$\vdots$ \\
$\vdots$
\end{tabular} & 른 & 菜 & 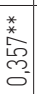 & 華 & 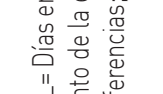 \\
\hline త্ & & & & & & & & & - & 草 & $\stackrel{\overbrace{}}{3}$ & 苦 & & $\frac{2}{5}$ & . & 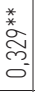 & $\stackrel{\bar{\Xi}}{\Xi}$ & 突 & 莕 & . & 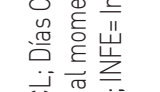 \\
\hline 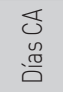 & & & & & & & & - & Е్. & $\begin{array}{l}\text { ¿े } \\
\text { ठ }\end{array}$ & है & 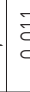 & & है & 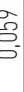 & $\begin{array}{l}\bar{\sigma} \\
\overline{0} \\
0\end{array}$ & $\frac{n_{0}^{*}}{\omega_{0}^{0}}$ & 응 & $\begin{array}{l}\text { ?. } \\
0 \\
0 \\
\end{array}$ & . & 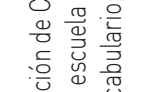 \\
\hline $\begin{array}{l}\text { T } \\
0 \\
0 \\
0 \\
\\
\end{array}$ & & & & & & 7 & & $\begin{array}{l}2 \\
2 \\
0 \\
0\end{array}$ & $\stackrel{2}{\stackrel{2}{0}}$ & 㫧 & 荥 & 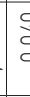 & & 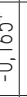 & $\begin{array}{c}2 \\
0 \\
0 \\
0\end{array}$ & 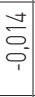 & . & 흐. & 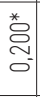 & 흐. & 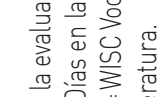 \\
\hline $\overrightarrow{0}$ & & & & & - & 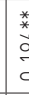 & & Ë & 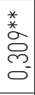 & 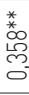 & 芴 & 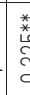 & & I & , & 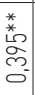 & 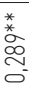 & $\begin{array}{l}2 \\
5 \\
0 \\
0\end{array}$ & 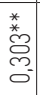 & $\begin{array}{l}\underbrace{*}_{*} \\
\stackrel{*}{*} \\
\tilde{\sigma}^{\circ}\end{array}$ & 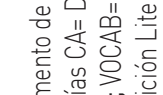 \\
\hline 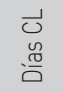 & & & & - & 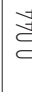 & 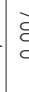 & & 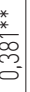 & $\begin{array}{l}\simeq \\
\text { o. } \\
0\end{array}$ & हे & : జ & z & & 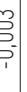 & $\frac{2}{6}$ & $\begin{array}{l}\infty \\
0 \\
0 \\
0 \\
1\end{array}$ & $\begin{array}{l}\text { ల్ } \\
\text { : }\end{array}$ & ? & $\begin{array}{l}\overline{0} \\
\overline{0} \\
0\end{array}$ & 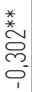 & 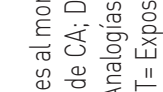 \\
\hline $\begin{array}{l}\bar{J} \\
0 \\
\tilde{D} \\
\end{array}$ & & & - & 념 & $\stackrel{*}{\stackrel{*}{5}}$ & 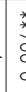 & & 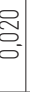 & 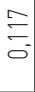 & $\begin{array}{l}\text { P }_{0}^{*} \\
\stackrel{0}{0}\end{array}$ & 薏 & $z$ & & יa & مُ & $\begin{array}{l}\text { oे } \\
\text { - } \\
\end{array}$ & $\underset{\Xi}{\sigma}$ & త్ & $\begin{array}{l}\stackrel{*}{*} \\
\frac{\sigma}{\sigma} \\
\sigma\end{array}$ & 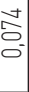 & 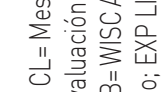 \\
\hline $\begin{array}{l}\text { 이 } \\
\text { व } \\
0 \\
0\end{array}$ & & - & $\begin{array}{l}\stackrel{*}{*} \\
\stackrel{\infty}{0} \\
\end{array}$ & 恙 & de & 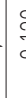 & & . & $\bar{E}$ & $\frac{\mathrm{m}}{0}$ & 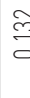 & 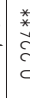 & & 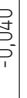 & $\frac{x^{*}}{5}$ & 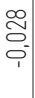 & 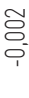 & 응 & Бे & . & 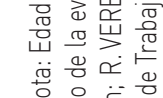 \\
\hline 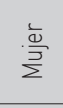 & - & $\frac{\mathrm{m}}{\mathrm{c}}$ & $\begin{array}{l}\text { 음 } \\
\text { o. } \\
\text { i }\end{array}$ & ప్ & ב & 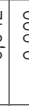 & & $\stackrel{8}{\circ}$ & 횽. & $\begin{array}{l}\text { o. } \\
0 \\
0 \\
\end{array}$ & 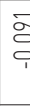 & 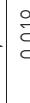 & & 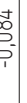 & है & 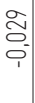 & 을 & 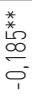 & 气̄ & 年 & 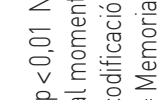 \\
\hline &. & $\begin{array}{l}0 \\
0 \\
0 \\
0\end{array}$ & $\begin{array}{l}0 \\
0 \\
0 \\
0 \\
\end{array}$ & \begin{tabular}{|l}
$\bar{c}$ \\
$u$ \\
.$\pi$ \\
$\frac{\pi}{c}$
\end{tabular} & $\bar{c}$ & 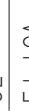 & & $\begin{array}{l}0 \\
0 \\
0 \\
0 \\
0\end{array}$ & $\overleftarrow{J}$ & 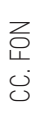 & 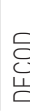 & 离 & & 倣 & $\frac{1}{2}$ & $\begin{array}{l} \\
\bar{\Sigma} \\
\\
\Sigma\end{array}$ & $\begin{array}{l}8 \\
\text { O } \\
ن \\
ن\end{array}$ & $\begin{array}{l}\underline{\underline{\underline{I}}} \\
\underline{\underline{u}} \\
\dot{u}\end{array}$ & 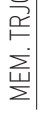 & 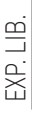 & 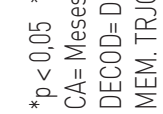 \\
\hline
\end{tabular}




\subsection{Variable dependiente CL}

El primer objetivo del estudio, determinar si los procesos superiores y las variables de conocimiento influyen en la CL de lectores muy incipientes, se abordó ingresando las variables superiores y de conocimiento junto con los controles y las variables de decodificación, en el mismo modelo de regresión para predecir la CL. El modelo de regresión explicó un 43,8\% de la varianza de la LC ( $F=10,404 ; g l=$ 14, 187; $p<0,01)$. La Tabla 3 muestra los resultados de la regresión con todas las variables estudiadas.

Tabla 3. Resultados regresión lineal con comprensión auditiva y comprensión de lectura como variables dependientes

\begin{tabular}{|c|c|c|c|c|c|c|c|c|c|c|}
\hline & \multicolumn{5}{|c|}{ Comprensión de lectura } & \multicolumn{5}{|c|}{ Comprensión auditiva } \\
\hline & \multirow[b]{2}{*}{$\mathrm{R}^{2}$} & \multirow[b]{2}{*}{$\mathrm{F}$} & \multicolumn{2}{|c|}{$\begin{array}{c}\text { Coef. no } \\
\text { estandarizados }\end{array}$} & \multirow[b]{2}{*}{ T } & \multirow[b]{2}{*}{$\mathrm{R}^{2}$} & \multirow[b]{2}{*}{$\mathrm{F}$} & \multicolumn{2}{|c|}{$\begin{array}{c}\text { Coef. no } \\
\text { estandarizados }\end{array}$} & \multirow[b]{2}{*}{$t$} \\
\hline & & & B & Error típ. & & & & B & Error típ. & \\
\hline & 0,438 & $10,404^{* *}$ & & & & 0,169 & $2,711^{* *}$ & & & \\
\hline Copago & & & $-0,058$ & 0,353 & $-0,164$ & & & $-8,026$ & 17.363 & -.462 \\
\hline Género (mujer) & & & $-0,088$ & 0,722 & $-0,122$ & & & 43,932 & 38.347 & 1.146 \\
\hline Días & & & 0,056 & 0,024 & $2,356^{*}$ & & & 1,007 & 1.840 & .547 \\
\hline Edad & & & 0,08 & 0,098 & 0,82 & & & 8,322 & 5.177 & 1.608 \\
\hline Decodificación & & & 1,329 & 0,449 & $2,959^{* *}$ & & & 17,301 & 23.660 & .731 \\
\hline CC. fonológica & & & 0,646 & 0,532 & 1,215 & & & $-25,612$ & 28.169 & -.909 \\
\hline Vocabulario & & & $-0,102$ & 0,119 & $-0,857$ & & & 1,883 & 6.300 & .299 \\
\hline $\begin{array}{l}\text { Memoria de } \\
\text { trabajo }\end{array}$ & & & 0,738 & 0,45 & 1,64 & & & 17,631 & 23.907 & .737 \\
\hline $\begin{array}{l}\text { Flexibilidad } \\
\text { cognitiva }\end{array}$ & & & 1,146 & 0,361 & $3,175^{* *}$ & & & 8,644 & 19.522 & .443 \\
\hline $\begin{array}{l}\text { Control } \\
\text { inhibitorio }\end{array}$ & & & $-1,327$ & 0,475 & $-2,794^{* *}$ & & & 30,214 & 25.122 & 1.203 \\
\hline Inferencias & & & 0,156 & 0,135 & 1,157 & & & 11,756 & 7.140 & 1.646 \\
\hline $\begin{array}{l}\text { Razonamiento } \\
\text { verbal }\end{array}$ & & & 0,364 & 0,153 & $2,380^{*}$ & & & 7,448 & 7.997 & .931 \\
\hline Monitoreo & & & 0,448 & 0,115 & $3,890^{* *}$ & & & 23,258 & 6.123 & $3.798^{* *}$ \\
\hline $\begin{array}{l}\text { Exposición a } \\
\text { libros }\end{array}$ & & & 0,572 & 0,152 & $3,765^{* *}$ & & & 9,302 & 8.008 & 1.162 \\
\hline
\end{tabular}

Se esperaba que la CL en estos decodificadores incipientes se viera explicada más que nada por elementos asociados a la decodificación y la conciencia fonológica, y no por los procesos 
cognitivos superiores o el conocimiento. Sin embargo, al contrario de lo esperado, se constató que varios de los procesos cognitivos superiores incluidos en el estudio contribuían a una varianza significativa en la comprensión lectora de los niños y niñas, incluso en este estadio de decodificación inicial. La regresión muestra que, además de la contribución de decodificación -que como se esperaba es significativa $(t=2.959 ; p<0,01)-$, también el monitoreo es significativo para predecir la CL $(t=3.890, p<0,01)$, y asimismo el razonamiento verbal $(t=2.380, p<0,05)$ y la exposición a libros $(t=$ $3.765 ; p<0,01)$. Estos efectos se observan aún después de controlar por funciones ejecutivas. Un resultado inesperado es la contribución de la variable control inhibitorio, que se asocia negativamente con la $\mathrm{CL}(t=-2.794 ; p<0,01)$.

\subsection{Variable dependiente CA}

Los resultados para CA son diferentes a los de CL. El modelo de regresión completo para la CA explicó solo un 16,9\% de la varianza ( $F$ $=3.169 ; g l=13,180 ; p<0,01)$. La Tabla 3 muestra los resultados de la regresión con todas las variables estudiadas. Se esperaba que al evaluar CA en $1^{\circ}$ básico esta fuera más fuertemente explicada por las habilidades cognitivas y lingüísticas complejas que la CL, pero esta predicción no se cumplió, ya que entre los procesos cognitivos complejos solo monitoreo tuvo una contribución significativa $(t=3.798, p<0,01)$, mientras que razonamiento verbal, inferencias y vocabulario no contribuyeron significativamente. Tampoco las habilidades de función ejecutiva, exposición a libros y vocabulario resultaron importantes, lo que es contrario a lo que se esperaba. Estos resultados podrían relacionarse con el hecho de que esta prueba fue fácil para los niños de $1^{\circ}$ básico, lo cual limitó la varianza explicada total.

\section{Discusión}

Los objetivos de este estudio fueron determinar si un conjunto de predictores cognitivos y lingüísticos complejos contribuía varianza a la CL y la CA en lectores incipientes, y comparar sus contribuciones a ambas. Estudios previos habían mostrado que estos predictores podían explicar varianza en la CL en lectores relativamente fluidos, 
pero no estaba claro que también contribuyeran a la CL en lectores novicios, y su estudio en la CA era limitado.

La CL demostró estar influenciada desde muy temprano por variables cognitivas superiores como el monitoreo de la propia comprensión y el razonamiento verbal, incluso tratándose de niños con muy lenta decodificación. Esto muestra que estas habilidades son necesarias para construir una representación coherente del discurso, desde los momentos más tempranos de la lectura de textos, e incluso pese a las demandas de memoria de trabajo impuestas por la falta de automatización en la lectura de palabras. Estos resultados apoyan la sugerencia de dar importancia a enseñar a los niños habilidades como la detección de inconsistencias, el monitoreo de la propia comprensión, y el razonamiento en torno a palabras y conceptos desde muy temprano y en forma explícita. La enseñanza de estas habilidades no debe relegarse a una etapa donde los niños puedan leer fluidamente textos de mediana complejidad, sino que debe priorizarse incluso desde la edad preescolar, ya que como muestran estos resultados, estas comenzarán a afectar la CL de los niños desde sus primeros intentos de comprender textos.

La habilidad de los niños para reconocer portadas de cuentos tuvo una fuerte influencia en la CL. Esto es consistente con la literatura de habla inglesa en relación con esta variable que muestra que los niños que crecen rodeados de libros se aproximan desde temprano naturalmente a la lectura, leen antes, adquieren más vocabulario y finalmente desarrollan mejor CL. Estos resultados destacan la necesidad de buscar estrategias para maximizar el acceso a libros especialmente de poblaciones vulnerables que no poseen estos recursos en sus hogares, y a aprovechar mejor los recursos de las bibliotecas escolares ya existentes en los establecimientos. El promedio de portadas reconocidas fue muy bajo, considerando que el instrumento incluyó títulos muy populares, cómics, y libros recomendados por el Mineduc para $1^{\circ}$ básico. Esto indica que los libros disponibles en los colegios no llegan a los niños en la dosis que se esperaría. 
Un resultado inesperado en la predicción de la CL fue que la variable control inhibitorio mostró una contribución negativa a la $\mathrm{CL}$ en $1^{\circ}$ básico, luego de controlar por las otras variables en el modelo. Esta variable fue medida con la Prueba de la cuerda floja, que mide la capacidad del niño de inhibir su velocidad "natural" al caminar, y caminar más lento de lo normal. Este resultado, aunque sorprendente, es consistente con un resultado previo obtenido con esta misma muestra en kínder (Strasser \& del Río, 2014) con una prueba diferente (golpe de lápiz). En ese estudio, la variable dependiente fue la comprensión de historias y se observó que, al controlar por las demás funciones ejecutivas, el control inhibitorio tenía un efecto negativo. Una posibilidad es que por tratarse de variables dependientes que se basan en el lenguaje expresivo (los niños deben proporcionar respuestas construidas a preguntas de comprensión literal e inferencial), una excesiva inhibición limite las respuestas de los niños y niñas, disminuyendo su puntaje. Esto es razonable si se considera que los modelos de regresión controlan las otras variables de función ejecutiva, tales como atención y memoria de trabajo, por lo que el efecto negativo de la inhibición no representa necesariamente que a mayor impulsividad menor CL, sino más bien que los niños que están en el extremo de la capacidad de inhibir sus respuestas predominantes, tienden a obtener menores puntajes.

En cuanto a la predicción de la CA, el modelo aquí aplicado explicó un porcentaje bajo de varianza. Entre las variables cognitivas y lingüísticas superiores, solo el monitoreo de la comprensión explicó una varianza significativa de la CA, subrayando la importancia de la metacognición para formar una representación coherente de un discurso, ya sea en modalidad escrita o auditiva. Sin embargo, las demás habilidades superiores -razonamiento verbal, inferencias, vocabulario- no predijeron varianza en la medida de CA. Es posible que esto se debiera a que los textos usados en la prueba fueran demasiado fáciles para los niños a esta edad, por lo que su comprensión no requería poner en juego estas habilidades complejas. Sin embargo, la prueba sí detectó la influencia de la variable monitoreo. Esto indica que la capacidad de monitorear la propia comprensión es tan importante que su contribución se manifestó incluso pese a las limitaciones metodológicas de la prueba. 
En resumen, se puede afirmar que las variables cognitivas superiores estudiadas resultan importantes en determinar la comprensión, en mayor medida de un texto escrito, pero también presentado en forma auditiva. El impacto de variables que permiten integrar el texto en un todo coherente se puede observar a partir de los momentos más incipientes de la decodificación y, por ende, es probable que esté presente incluso en la etapa prelectora. Esto es consistente con lo encontrado por Florit y colegas (Florit et al., 2011; Florit et al., 2009; Florit et al., 2014) y por Strasser y del Río (2014) en niños prelectores.

En general, las dos variables más predictivas para la CL resultaron ser monitoreo y exposición a textos escritos, siendo la primera la única en tener influencia tanto en la CL como en la CA. Llama la atención que inferencias y vocabulario no hayan resultado significativos para ninguna de las dos formas de comprensión, ya que en otras investigaciones se ha visto que estas habilidades se relacionan con CL o con la CA (Cain et al., 2004; Oakhill \& Cain, 2012; Strasser y del Río, 2013). La colinealidad entre estas variables y otras como analogías (razonamiento verbal) puede explicar en parte este hecho.

En cuanto a sus implicancias prácticas, estos resultados enfatizan la importancia de trabajar en el aula las habilidades cognitivas complejas desde temprano, en forma tan sistemática como se trabaja la decodificación. En este sentido, llama la atención que los días transcurridos en el año escolar solo afecten a la CL y no a la CA, lo que sugiere que la CA es menos trabajada en el contexto escolar. Los resultados de esta investigación apoyan la idea de que se le debe dar un espacio específico en la escuela a la oralidad, donde estas habilidades sean enseñadas y estimuladas antes y durante el tiempo en que los niños están trabajando en convertirse en lectores fluidos.

A futuro será necesario llevar a cabo investigaciones que incorporen más variables de vocabulario (profundidad y amplitud), de conocimiento de la estructura de las historias, así como mejores pruebas de inferencias, que permitan alcanzar un modelo más completo de comprensión que guíe en el desarrollo de mejores estrategias prácticas para la enseñanza de la lectura y la comprensión 
en general. Asimismo, medir la comprensión del discurso en formatos no escritos es un desafío en esta edad, como pudo verse por las dificultades metodológicas de la prueba. Mejorar la evaluación de estos importantes constructos puede contribuir a una mejor enseñanza de ellos, en tanto los docentes podrían identificar mejor las áreas de debilidades y subcomponentes que se puedan entrenar.

\section{Referencias}

Aikens, N. L. \& Barbarin, O. (2008). Socioeconomic differences in reading trajectories: The contribution of family, neighborhood, and school contexts. Journal of Educational Psychology, 100, 235-251. http://dx. doi. org/10.1037/0022-0663.100.2.235

Andreassen, R. \& Braten, I. (2010). Examining the prediction of reading comprehension on different multiple choice tests. Journal of Research in Reading, 33, 263-283. http://dx.doi.org/10.1111/j.14679817.2009.01413.x

Beck, I. L., McKeown, M. G., \& Kucan, L. (2002). Bringing words to life. Robust vocabulary instruction. New York: Guilford Press.

Berninger, V. W., Abbott, R. D., Swanson, H. L., Lovitt, D., Trivedi, P., Lin, S., ... \& Amtmann, D. (2010). Relationship of word- and sentence-level working memory to reading and writing in second, fourth, and sixth grade. Language, Speech, and Hearing Services in Schools, 41, 179-193. http://dx.doi.org/10.1044/0161-1461(2009/08-0002)

Cain, K. (2006). Individual differences in children's memory and reading comprehension: An investigation of semantic and inhibitory deficits. Memory, 14, 553-569. http://dx.doi.org/10.1080/09658210600624481

Cain, K. \& Oakhill, J. (2006). Profiles of children with specific reading comprehension difficulties. British Journal of Educational Psychology, 76, 683-696. http://dx.doi.org/10.1348/000709905X67610

Cain, K., Oakhill, J., \& Bryant, P. (2004). Children's reading comprehension ability: Concurrent prediction by working memory, verbal ability, and component skills. Journal of Educational Psychology, 96, 31-42. http:// dx.doi.org/10.1037/0022-0663.96.1.31

Canet-Juric, L., Burin, D., Andrés, M. L., y Urquijo, S. (2013). Perfil cognitivo de niños con rendimientos bajos en comprensión lectora. Anales de Psicología, 29, 996-1005. http://dx.doi.org/10.6018/ analesps.29.3.138221 
Catts, H. W. \& Kamhi, A. G. (Eds.). (2005). Language and reading disabilities ( $2^{\text {nd }}$ ed.). Boston: Pearson Education, Inc.

Davidse, N. J., de Jong, M. T., Bus , A. G., Huijbregts, S. C. J., \& Swaab, H. (2011). Cognitive and environmental predictors of early literacy skills. Reading \& Writing, 24, 395-412. http://dx.doi.org/10.1007/ s11145-010-9233-3

Defior-Citorel, S., Fonseca, L., Gottheil, B., Aldrey, A., Jiménez Fernández, G., Pujals, M., Rosa, G., \& Serrano Chica, F. D. (2006). LEE. Test de Lectura y Escritura en Español. Buenos Aires: Paidós.

Eason, S. H., Goldberg, L. F., Young, K. M., Geist, M. C., \& Cutting, L. E. (2012). Reader-text interactions: How differential text and question types influence cognitive skills needed for reading comprehension. Journal of Educational Psychology, 104, 515-528. http://dx.doi. org/10.1037/a0027182

Florit, E., Roch, M., \& Levorato, M. C. (2011). Listening text comprehension of explicit and implicit information in ireschoolers: The role of verbal and inferential skills. Discourse Processes, 48, 119-138. http://dx.doi. org/10.1080/0163853X.2010.494244

Florit, E., Roch, M., \& Levorato, M. C. (2014). Listening text comprehension in preschoolers: A longitudinal study on the role of semantic components. Reading and Writing, 27, 793-817. http://dx.doi.org/10.1007/s11145013-9464-1

Florit, E., Roch, M., Altoè, G., \& Levorato, M. C. (2009). Listening comprehension in preschoolers: The role of memory. British Journal of Developmental Psychology, 27, 935-951. http://dx.doi. org/10.1348/026151008X397189

Garner, J. K. \& Bochna, C. R. (2004). Transfer of a listening comprehension strategy to independent reading in first-grade students. Early Childhood Education Journal, 32, 69-74. http://dx.doi.org/10.1007/s10643-0041071-y

Hoover, W. A. \& Gough, P. B. (1990). The simple view of reading. Reading E. Writing, 2, 127-160. http://dx.doi.org/10.1007/BF00401799

Kendeou, P., Rapp, D. N., \& van den Broek, P. (2003). The influence of reader's prior knowledge on text comprehension and learning from text. En R. Nata (Ed.), Progress in Education, Vol.13 (pp. 189-209). New York: Nova Science Publishers, Inc.

Kendeou, P., van den Broek, P., White, M. J., \& Lynch, J. S. (2009). Predicting reading comprehension in early elementary school: The independent contributions of decoding and oral language skills. Journal of Educational Psychology, 101, 765-778. http://dx.doi.org/10.1037/a0015956 
Kochanska, G., Murray, K., Jacques, T. Y., Koenig, A. L., \& Vandegeest, K. A. (1996). Inhibitory control in young children and its role in emerging internalization. Child Development, 67, 490-507. http://dx.doi. org/10.2307/1131828

Lehto, J. E. \& Anttila, M. (2003). Listening comprehension in primary level grades two, four, and six. Scandinavian Journal of Educational Research, 47, 133-143. http://dx.doi.org/10.1080/00313830308615

Manzi, J., Strasser, K., San Martín, E., \& Contreras, D. (2008). Quality of education in Chile. Santiago: MideUC-Centro de Medición de la Pontificia Universidad Católica de Chile.

Mizala, A. \& Torche, F. (2012). Bringing the schools back in: The stratification of educational achievement in the Chilean voucher system. International Journal of Educational Development, 32, 132-144. http://dx. doi. org/10.1016/j.ijedudev.2010.09.004

Mol, S., Bus, A., \& de Jong, M. (2009). Interactive book reading in early education: A tool to stimulate print knowledge as well as oral language. Review of Educational Research, 79, 979-1007. http://dx.doi. org/10.3102/0034654309332561

Mol, S., Bus, A., de Jong, M., \& Smeets, D. (2008). Added value of dialogic parent-child book readings: A meta-analysis. Early Education and Development, 19, 7-29. http://dx.doi.org/10.1080/10409280701838603

Muñoz-Sandoval, A. F., Woodcock, R. W., McGrew, K. S., \& Mather, N. (2005). Batería III Woodcock-Muñoz. Itasca, IL: Riverside Publishing.

Nation, K., Cocksey, J., Taylor, J. S. H., \& Bishop, D. V. M. (2010). A longitudinal investigation of early reading and language skills in children with poor reading comprehension. Journal of Child Psychology and Psychiatry, 51, 1031-1039. http://dx.doi.org/10.1111/j.14697610.2010.02254.x

Oakhill, J. V. (1982). Constructive processes in skilled and less skilled comprehenders' memory for sentences. British Journal of Psychology, 73, 13-20. http://dx.doi.org/10.1111/j.2044-8295.1982.tb01785.x

Oakhill, J. V. \& Cain, K. (2012). The precursors of reading ability in young readers: Evidence from a four-year longitudinal study. Scientific Studies of Reading, 16, 91-121. http://dx.doi.org/10.1080/10888438.2010.529219

Oakhill, J., Hartt, J., \& Samols, D. (2005). Levels of comprehension monitoring and working memory in good and poor comprehenders. Reading and Writing, 18, 657-686. http://dx.doi.org/10.1007/s11145-005-3355-z

Organisation for Economic Co-operation and Development, OECD. (2012). Equity and quality in education: Supporting Disadvantaged Students and Schools. http://dx.doi.org/10.1787/9789264130852-en 
Ouellette, G. \& Beers, A. (2010). A not-so-simple view of reading: how oral vocabulary and visual-word recognition complicate the story. Reading and Writing, 23, 189-208. http://dx.doi.org/10.1007/s11145-0089159-1

Petersen, D. B. \& Spencer, T. D. (2012). The narrative language measures: Tools for language screening, progress monitoring, and intervention planning. Perspectives on Language Learning and Education, 19, 119-129. http://dx.doi.org/10.1044/1le19.4.119

Pickering, S. J. \& Gathercole, S. E. (2001). Working memory test battery for children. London: Psychological Corp.

Potocki, A., Ecalle, J., \& Magnan, A. (2013). Narrative comprehension skills in 5-year-old children: Correlational analysis and comprehender profiles. The Journal of Educational Research, 106, 14-26. http://dx.doi.org/10.1 080/00220671.2012.667013

Ramírez, V. y Rosas, R., (2007). Estandarización del WISC-III en Chile: descripción del Test, estructura factorial y consistencia interna de las escalas. Psykhe, 16, 91-109. http://dx.doi.org/10.4067/S071822282007000100008

Strasser, K. \& del Río, F. (2014). The role of comprehension monitoring, theory of mind and vocabulary depth in predicting story comprehension and recall of kindergarten children. Reading Research Quarterly, 49(2), 169187. http://dx.doi.org/10.1002/rrq.68

Tighe, E. L. \& Schatschneider, C. (2014). A dominance analysis approach to determining predictor importance in third, seventh, and tenth grade reading comprehension skills. Reading and Writing, 27, 101-127. http:// dx.doi.org/10.1007/s11145-013-9431-x

Tilstra, J., McMaster, K., Van den Broek, P., Kendeou, P., \& Rapp, D. (2009). Simple but complex: Components of the simple view of reading across grade levels. Journal of Research in Reading, 32, 4, 383-401. http://dx.doi. org/10.1111/j.1467-9817.2009.01401.x

Unesco (2013). Tercer estudio regional comparativo y explicativo Terce. Análisis curricular. Oficina Regional de Educación para América Latina y el Caribe. Santiago de Chile: Orealc/Unesco, Santiago.

Willoughby, M. T., Wirth, R. J., \& Blair, C. B. (2012). Executive function in early Childhood: Longitudinal measurement invariance and developmental change. Psychological Assessment, 24(2), 418-431. http:// dx.doi.org/10.1037/a0025779

Recibido: 16/10/2015

Aceptado: 17/05/2016 